\title{
Quantitative trait locus analysis of parasitoid counteradaptation to symbiont-conferred resistance
}

\author{
Gabriel F. Ulrich $\mathbb{B}^{1,2} \cdot$ Niklaus Zemp $\mathbb{B}^{3} \cdot$ Christoph Vorburger $\mathbb{B}^{1,2} \cdot$ Hélène Boulain $\mathbb{D}^{1,4}$ \\ Received: 23 November 2020 / Revised: 26 April 2021 / Accepted: 27 April 2021 / Published online: 19 May 2021 \\ (c) The Author(s) 2021. This article is published with open access
}

\begin{abstract}
Insect hosts and parasitoids are engaged in an intense struggle of antagonistic coevolution. Infection with heritable bacterial endosymbionts can substantially increase the resistance of aphids to parasitoid wasps, which exerts selection on parasitoids to overcome this symbiont-conferred protection (counteradaptation). Experimental evolution in the laboratory has produced counteradapted populations of the parasitoid wasp Lysiphlebus fabarum. These populations can parasitize black bean aphids (Aphis fabae) protected by the bacterial endosymbiont Hamiltonella defensa, which confers high resistance against $L$. fabarum. We used two experimentally evolved parasitoid populations to study the genetic architecture of the counteradaptation to symbiont-conferred resistance by QTL analysis. With simple crossing experiments, we showed that the counteradaptation is a recessive trait depending on the maternal genotype. Based on these results, we designed a customized crossing scheme to genotype a mapping population phenotyped for the ability to parasitize Hamiltonellaprotected aphids. Using 1835 SNP markers obtained by ddRAD sequencing, we constructed a high-density linkage map consisting of six linkage groups (LGs) with an overall length of $828.3 \mathrm{cM}$ and an average marker spacing of $0.45 \mathrm{cM}$. We identified a single QTL associated with the counteradaptation to Hamiltonella in L. fabarum on linkage group 2. Out of 120 genes located in this QTL, several genes encoding putative venoms may represent candidates for counteradaptation, as parasitoid wasps inject venoms into their hosts during oviposition.
\end{abstract}

\section{Introduction}

Interactions between organisms are important drivers of evolution. Among them, host-parasite interactions represent particularly intimate relationships between species (Windsor 1998). In this context, reciprocal selection can lead to

Supplementary information The online version contains supplementary material available at https://doi.org/10.1038/s41437021-00444-7.

Hélène Boulain

helene.boulain@unil.ch

1 EAWAG, Swiss Federal Institute of Aquatic Science and Technology, Überlandstrasse 133, 8600 Dübendorf, Switzerland

2 Institute of Integrative Biology, ETH Zürich, Universitätsstrasse 16, 8092 Zürich, Switzerland

3 Genetic Diversity Centre, Department of Environmental Systems Sciences, ETH Zürich, 8092 Zürich, Switzerland

4 Present address: Department of Ecology and Evolution, University of Lausanne, 1015 Lausanne, Switzerland antagonistic coevolution through dynamic adaptation and counteradaptation, often compared to an arms race between species (Van Valen 1977; Woolhouse et al. 2002). Classical models of host-parasite coevolution treat resistance and infectivity as traits encoded by the genome of the host and parasite, respectively (Anderson and May 1982; Frank 1997; Sasaki 2000). While this is often the case (e.g., Flor 1956; Dubuffet et al. 2007; Bento et al. 2017), there are also numerous examples where these traits are influenced or even determined by symbionts associated with hosts or parasites. For example, the human gut microbiome is an important determinant of susceptibility to pathogens (Bäumler and Sperandio 2016), microbial endosymbionts can increase insect resistance to various types of parasites (Oliver et al. 2014), and even macrobial symbionts like tending ants can reduce parasitism of honeydew-producing insects (Itioka and Inoue 1996). Similarly, parasites may be aided by symbionts for successful infection, as in the case of insect-parasitic nematodes and their symbiotic Xenorhabdus and Photorhabdus bacteria (Goodrich-Blair and Clarke 2007), or in parasitic wasps aided by viral symbionts (Coffman and Burke 2020). 
In the case of maternally transmitted endosymbionts protecting hosts against parasitism (Haine 2008), the symbiont's contribution to host resistance is heritable and responsive to selection by parasites (Jaenike 2012). As a result, the reciprocal adaptation in host-parasite coevolution becomes mediated by symbionts (reviewed in Vorburger and Perlman 2018). For these adaptive changes to happen, genetic variation is required in both parasite and host populations. The advent of next-generation sequencing combined with laboratory-based experiments manipulating host-parasite systems has allowed to investigate this genetic diversity and address the genomic basis underpinning the mechanisms of coevolution (Schlötterer et al. 2015).

Parasitoids form a peculiar group within parasites as they inevitably kill their hosts upon completion of their life cycle (Godfray 1994). Parasitoids thus tend to have more severe impacts on host fitness than parasites, imposing strong selection on the host to resist. The large majority of parasitoid insects belongs to the order Hymenoptera, which comprises at least several hundred thousand species of parasitoid, accounting for $>75 \%$ of the order's species richness (Heraty 2009; Forbes et al. 2018). While idiobiont parasitoids kill or paralyze the host during oviposition to arrest host development, koinobiont parasitoids allow their host to continue development during parasitism (Quicke 2015). Hence, koinobiont endoparasitoids are exposed to and must bypass the host's immune system while keeping it functional to prevent the host from succumbing to opportunistic infections. During koinobiont evolution, various strategies have therefore been selected to finely manipulate the host's immune defenses (Pennacchio and Strand 2006; Burke and Strand 2014).

Aphids and their associated endoparasitoid wasps provide a unique system to study host-parasite coevolution as, in many cases, the outcome of their interaction is also influenced by facultative bacterial endosymbionts of aphids (Oliver et al. 2003). The vertically transmitted Hamiltonella defensa (Enterobacteriaceae) is among the most widespread of these symbionts (Oliver et al. 2003; Moran et al. 2005) being found in $41 \%$ out of 131 examined aphid species (Henry et al. 2015). It confers significant protection against parasitoid wasps in multiple aphid species (reviewed in Oliver et al. 2014; Vorburger 2014). The protection is associated with the presence of the APSE bacteriophage in H. defensa's genome (Oliver et al. 2009). Indeed, APSE carry strain-specific "cassettes" encoding toxins likely interfering with parasitoid development (Oliver et al. 2009). However, resistance against parasitoids is not only attributable to the symbiont-bacteriophage association aphids carry, as different symbiont-free aphid genotypes also differ in their resistance (Sandrock et al. 2010; Martinez et al. 2014). The variation in aphid susceptibility to parasitism, along with $H$. defensa strain-specific strength of protection, may explain natural variation observed in aphid-wasp interactions (Oliver et al. 2005; McLean and Godfray 2015; Oliver and Higashi 2019). Moreover, there is also ample genetic variation in wasp infectivity (Schmid et al. 2012; Vorburger and Rouchet 2016).

This natural variation is particularly well described in the interaction between the black bean aphid Aphis fabae (Aphididae) and the koinobiont parasitoid wasp Lysiphlebus fabarum (Braconidae), for which significant genotype-bygenotype interactions occur between wasp genotypes and $H$. defensa strains protecting their aphid hosts (Schmid et al. 2012; Cayetano and Vorburger 2013). A first experimental evolution study with wild-collected $L$. fabarum populations demonstrated rapid and strain-specific adaptation of parasitoids to Hamiltonella-protected aphids, likely due to standing genetic variation in natural populations (Rouchet and Vorburger 2014). A follow-up study also employing experimental evolution confirmed these results and compared patterns of gene expression between Hamiltonellaadapted parasitoids and controls using transcriptomics (Dennis et al. 2017). Starting from a genetically diverse initial wasp population, replicated subpopulations were evolved on a single $A$. fabae genotype harboring either the $H$. defensa strain H76, the $H$. defensa strain H402, or no defensive symbiont (control). While symbiont-conferred resistance was high at the beginning, wasps facing Hamiltonella-protected aphids exhibited significant counteradaptation (i.e., evolved ability to parasitize protected hosts) already after ten generations, while control wasps remained poorly able to parasitize aphids harboring $H$. defensa. Putative venom genes and virus-associated genes were overrepresented among the differentially expressed genes between Hamiltonella-adapted and control populations, suggesting a role of these genes in the counteradaptation (Dennis et al. 2017). Despite these findings, the genetic architecture and the molecular mechanisms underlying the counteradaptation remain unclear. Moreover, the contributions of larval and maternal genotypes to the counteradapted phenotype are yet to be determined.

Both maternal and larval traits can influence parasitism success in parasitoid wasps (Burke and Strand 2014). Maternal behavior may play a role in parasitoid counteradaptation, since preference for younger hosts (Schmid et al. 2012) and self-superparasitism, that is depositing more than one egg per host (Oliver et al. 2012), were both shown to affect reproductive success of wasps on protected hosts. However, there was no evidence that these traits evolved in the study by Dennis et al. (2017). Other maternal factors like venoms, polydnaviruses, and virus-like particles, which many parasitoid wasp species inject into the host alongside their eggs to circumvent host defenses, may also be involved in counteradaptation (Moreau and Asgari 2015; Dennis et al. 2017; Drezen et al. 2017). Larval traits, such as 
Table 1 Prediction of female offspring survival and reproduction in experimental crosses of evolved Lysiphlebus fabarum populations under three different hypotheses.

\begin{tabular}{|c|c|c|c|c|c|c|}
\hline \multirow{2}{*}{$\begin{array}{l}\begin{array}{l}\text { Parental } \\
\text { genotypes }\end{array} \\
\text { ○ }\end{array}$} & \multirow{2}{*}{$\begin{array}{l}\begin{array}{l}\text { Offspring } \\
\text { genotypes }^{\mathrm{a}}\end{array} \\
\text { ○ }\end{array}$} & \multicolumn{3}{|c|}{$\begin{array}{l}\text { Female offspring survival on } \mathrm{H}+\text { aphids } \\
\text { (first generation) }\end{array}$} & \multicolumn{2}{|c|}{$\begin{array}{l}\text { Virgin RS female } \\
\text { reproduction on } \mathrm{H}+\text { aphids } \\
\text { (second generation) }\end{array}$} \\
\hline & & $\begin{array}{l}\text { H1: larval } \\
\text { dominant }\end{array}$ & $\begin{array}{l}\mathrm{H} 2 \text { : larval } \\
\text { recessive }\end{array}$ & $\begin{array}{l}\mathrm{H} 3: \\
\text { maternal }\end{array}$ & $\begin{array}{l}\text { H3.1: } \\
\text { dominant }\end{array}$ & $\begin{array}{l}\mathrm{H} 3.2 \text { : } \\
\text { recessive }\end{array}$ \\
\hline $\mathrm{RR} \times \mathrm{R}$ & RR R & $\mathrm{Y}$ & $\mathrm{Y}$ & $\mathrm{Y}$ & na & na \\
\hline $\mathrm{RR} \times \mathrm{S}$ & RS R & $\mathrm{Y}$ & $\mathrm{N}$ & $\mathrm{Y}$ & $\mathrm{Y}$ & $\mathrm{N}$ \\
\hline $\mathrm{SS} \times \mathrm{R}$ & RS S & $\mathrm{Y}$ & $\mathrm{N}$ & $\mathrm{N}$ & $\mathrm{Y}$ & $\mathrm{N}$ \\
\hline $\mathrm{SS} \times \mathrm{S}$ & SS S & $\mathrm{N}$ & $\mathrm{N}$ & $\mathrm{N}$ & na & na \\
\hline
\end{tabular}

$R$ resistant population, $S$ susceptible population, $Y$ yes (adult wasps emerged from mummified aphids), $N$ no (no adult wasps emerged from mummified aphids), na not applicable.

${ }^{a}$ Females are diploid and males are haploid. toxins or teratocyte excretion, may as well contribute to the counteradapted phenotype (Burke and Strand 2014).

Taking advantage of the recently published genome of $L$. fabarum (Dennis et al. 2020) and the continued availability of the experimentally evolved populations created by Dennis et al. (2017), we conducted a quantitative trait locus (QTL) study to investigate the genomic architecture of the counteradaptation to $H$. defensa in L. fabarum wasps. We first characterized the general nature of the counteradaptation trait (maternal vs larval determination, dominant vs recessive inheritance) with crossing experiments followed by parasitism bioassays. Based on these results, we designed a crossing scheme for high-density linkage map construction and QTL mapping. We used ddRAD sequencing for genotyping and measured parasitism success on Hamiltonella-protected hosts (offspring counts) as our phenotypic measure of parasitoid counteradaptation.

\section{Materials and methods}

\section{Host and parasitoid lines}

Black bean aphids ( $A$. fabae) were reared on their host plant Vicia faba (Fabaceae) in a climate chamber at $22{ }^{\circ} \mathrm{C}$ with a 16-h photoperiod to ensure clonal reproduction. Two sublines of A. fabae clone A06-407 were used: the original A06-407 clone, which was free of any known defensive endosymbionts, and the modified A06-407 clone harboring the $H$. defensa strain H76 (Vorburger et al. 2009; Dennis et al. 2017). The original ( $H$. defensa negative) and the modified $(H$. defensa positive, harboring the $H$. defensa strain $\mathrm{H76}$ ) aphid lines are in the following called $\mathrm{H}-$ and $\mathrm{H}+$, respectively.

We used two experimentally evolved populations of the parasitoid wasp L. fabarum. One was adapted to the presence of Hamiltonella in host aphids, the other was not. Wasp populations were established by Dennis et al. (2017) from a mixture of nine collections of sexually reproducing, haplo-diploid L. fabarum from six locations across Switzerland. Experimental evolution was conducted by rearing wasps exclusively on $\mathrm{H}-$ or $\mathrm{H}+$ aphids, leading to counteradaptation in the $\mathrm{H}+$ treatment; wasps reared on $\mathrm{H}+$ aphids evolved an improved ability to parasitize $\mathrm{H}+$ aphids compared to wasps reared on $\mathrm{H}$ - aphids (see Dennis et al. 2017 for more details). After maintaining treatments for 24 generations in 4 replicate populations each, replicates were combined and treatments were continued unreplicated at a population size of 200 individuals (see Rossbacher and Vorburger 2020 for details). Until the onset of the experiments presented here, parasitoid populations had been reared for approximately 140 generations on either $\mathrm{H}-$ or $\mathrm{H}+$ aphids (since September 2013). At this point, the population reared on $\mathrm{H}+$ aphids was able to parasitize $\mathrm{H}+$ aphids nearly as well as $\mathrm{H}$ - aphids, whereas the population reared on $\mathrm{H}$ - aphids was only able to parasitize $\mathrm{H}-$ aphids but not $\mathrm{H}+$ aphids. In the following, we refer to the wasp population adapted to $\mathrm{H}+$ aphids as $\mathrm{R}$ (= and to the population adapted to $\mathrm{H}-$ aphids as $\mathrm{S}$ (= ceptible to Hamiltonella).

\section{Experiment 1: characterization of general inheritance patterns}

To determine whether the evolved ability to parasitize $\mathrm{H}+$ aphids is mainly determined by the larval or the maternal genotype, and whether it shows a dominant or recessive inheritance pattern, crossing experiments were combined with no-choice bioassays over two generations of wasps. In the first generation, all possible combinations of males and females from the $\mathrm{R}$ and $\mathrm{S}$ populations were crossed in order to quantify their ability to reproduce on $\mathrm{H}+$ aphids (Table 1). Assuming that this ability is governed by a single Mendelian locus with two alleles ( $\mathrm{R}$ and $\mathrm{S}$ ), which are fixed in the respective populations (likely an oversimplification), allowed us to postulate three mutually exclusive hypotheses (H1-H3) that make different predictions for the outcome of these crosses (Table 1). To indicate genotypes and ploidy, crosses 
are depicted in the following as, e.g., $\mathrm{RR} \times \mathrm{S}$, meaning that a (diploid) female from the $\mathrm{R}$ population was crossed with a (haploid) male from the $\mathrm{S}$ population.

(H1) The counteradaptation is larval and dominant. Under $\mathrm{H} 1, \mathrm{RR} \times \mathrm{R}, \mathrm{RR} \times \mathrm{S}$, and $\mathrm{SS} \times \mathrm{R}$ crosses are expected to produce female offspring on $\mathrm{H}+$ aphids, as homozygous $\mathrm{RR}$ and heterozygous RS female larvae would be of the R phenotype and thus counteradapted. Homozygous SS daughters from $\mathrm{SS} \times \mathrm{S}$ crosses would fail to develop. If the counteradaptation was larval but inherited in an intermediate rather than dominant fashion, the expectation remains the same as under $\mathrm{H} 1$, albeit with the possibility that $\mathrm{RR} \times \mathrm{S}$ and $\mathrm{SS} \times \mathrm{R}$ crosses produce fewer female offspring than $\mathrm{RR} \times \mathrm{R}$ crosses.

(H2) The counteradaptation is larval and recessive. Under $\mathrm{H} 2$, only $\mathrm{RR} \times \mathrm{R}$ crosses would produce female offspring on $\mathrm{H}+$ aphids. $\mathrm{RR} \times \mathrm{S}, \mathrm{SS} \times \mathrm{R}$, and $\mathrm{SS} \times \mathrm{S}$ crosses are expected to not produce any female offspring as their heterozygous (RS) or homozygous (SS) daughters would be of the $\mathrm{S}$ phenotype and thus not counteradapted.

(H3) The counteradaptation is maternal. Under H3, the $\mathrm{RR} \times \mathrm{R}$ and $\mathrm{RR} \times \mathrm{S}$ crosses are expected to produce female offspring and the $\mathrm{SS} \times \mathrm{R}$ and $\mathrm{SS} \times \mathrm{S}$ crosses are not, as the genotype of the mother is decisive for offspring survival. If both maternal and larval effects were at play, the sex ratio in offspring from the $\mathrm{RR} \times \mathrm{S}$ crosses is expected to be male biased compared to $\mathrm{RR} \times \mathrm{R}$ crosses, due to a disadvantage of RS larvae compared to RR larvae, while haploid male larvae have an $\mathrm{R}$ genotype in either case.

To isolate wasps prior to use in experiments, mummies (parasitized aphids approaching parasitoid emergence) were collected and stored individually in $1.5 \mathrm{ml}$ Eppendorf tubes. Thus, adult wasps had never encountered another wasp or aphid before (naive virgins). Zero-to-3 days after hatching, the wasps were paired and given 20-120 min for mating in $1.5 \mathrm{ml}$ Eppendorf tubes. Although there was no control whether mating occurred in the given amount of time, mating was usually observed within the first $30 \mathrm{~s}$ of having wasp pairs in the same tube. Then the wasps were released on a caged plant with an aphid colony consisting of a known number of 0-48h-old $\mathrm{H}+$ aphid nymphs. The mean \pm standard deviation (SD) number of aphid nymphs provided per cross was $43.5 \pm 14.9$. Adult wasps were removed from colonies $24 \mathrm{~h}$ after release. Nine days after adding wasps, plants were enclosed in cellophane bags and left to dry out at $22{ }^{\circ} \mathrm{C}$ for hatching and subsequent sexing and counting of wasp offspring. Differences in numbers of female offspring between the different crosses of the first generation were analyzed with Mann-Whitney $U$ tests. A generalized linear model (GLM) was used to analyze differences in sex ratios. Statistical analyses were performed using $\mathrm{R}$ version 3.5.2 (R Core Team 2018).

Because findings from the first generation of crosses supported H3 (see "Results"), two extensions of H3 (H3.1 and H3.2) were tested in a second generation of crosses to determine whether the maternal counteradaptation was dominant or recessive (Table 1). To this end, we tested the ability of 20 virgin female offspring from $10 \mathrm{RR} \times \mathrm{S}$ crosses (i.e., heterozygous $\mathrm{RS}$ females) to reproduce on $\mathrm{H}+$ aphids. The mean \pm SD number of aphid nymphs provided per RS female was $21.9 \pm 9.5$.

(H3.1) The counteradaptation is maternal and dominant. Under H3.1, RS females are expected to reproduce successfully on $\mathrm{H}+$ aphids, because they are of the $\mathrm{R}$ phenotype. They are expected to produce only male offspring as they are virgins (arrhenotokous parthenogenesis). This scenario is indistinguishable from cytoplasmic inheritance, which would require further examination.

(H3.2) The counteradaptation is maternal and recessive. Under H3.2, RS females are not expected to reproduce on $\mathrm{H}+$ aphids, because they are of the $\mathrm{S}$ phenotype.

\section{Experiment 2: crosses and phenotyping for QTL study}

To obtain a mapping population and phenotype data, a crossing scheme similar to the one by Pannebakker et al. (2011) was realized (Fig. 1). The crossing design relied on two main assumptions: First, we assumed that the alleles responsible for the counteradaptation are fixed in alternative states in the $\mathrm{R}$ and $\mathrm{S}$ populations. Second, due to the findings from the first experiment, we assumed the counteradaptation to be recessive and determined by the maternal genotype (see "Results"). In the first generation (P generation), a single $\mathrm{S}$ female was crossed with an $\mathrm{R}$ male to produce heterozygous female RS offspring ( $\mathrm{F} 1$ generation). $\mathrm{F} 1$ females were allowed to reproduce as naive virgins to produce a recombinant male-only mapping population $(\mathrm{F} 2$ generation, Fig. 1A). F2 males were then backcrossed into the $\mathrm{R}$ background (each male with one $\mathrm{RR}$ female) to produce F3 female offspring for phenotyping (Fig. 1B). All reproduction up to the emergence of $\mathrm{F} 3$ females took place on $\mathrm{H}-$ aphids (Fig. 1) to avoid any selection. P individuals, $\mathrm{F} 1$ females and F2 males were stored in $1.5 \mathrm{ml}$ Eppendorf tubes at $-80^{\circ} \mathrm{C}$ for subsequent genotyping.

Phenotyping was conducted by letting naive virgin F3 females oviposit for $24 \mathrm{~h}$ on colonies with a known number of approximately 24-72-h-old $\mathrm{H}+$ aphid nymphs and subsequently counting their offspring as previously described. The average \pm SD number of aphid nymphs provided was $40.9 \pm 13.6$. Wasps were added to the aphid colonies in an open Eppendorf tube. If possible, two sister F3 females from the same recombinant F2 father were added to each aphid colony in order to reduce the occurrence of false negatives, i.e., random failures to reproduce that are unrelated to the females' genotype, e.g., due to harmful handling or death before oviposition. F3 sister females are identical 


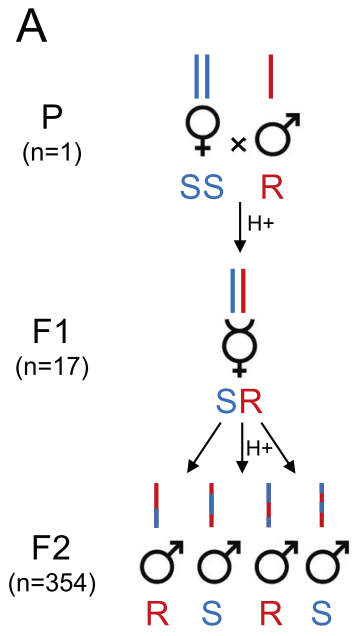

Fig. 1 Experimental crossing procedure for QTL analysis. Crossing design used to obtain a F2 mapping population for genotyping (A) and a F3 population for phenotyping (B). In a first step, two $\mathrm{P}$ generation individuals (parents), a diploid female from the symbiontsusceptible population, and a haploid male from the symbiont-resistant population were crossed to obtain 17 heterozygous F1 hybrid females. F1 hybrid females were allowed to reproduce as virgins-i.e., arrhenotokous parthenogenesis-to obtain 354 recombinant F2 males (mapping population), which were either carrying the $\mathrm{S}$ (susceptible)

concerning their paternal chromosome set and share the same $\mathrm{R}$ population background concerning their maternal chromosome set. They are considered clonal sibships (Pannebakker et al. 2011).

The phenotype we measured was the number of wasp offspring produced per $\mathrm{H}+$ aphid colony. This measure exhibited strong variation and zero inflation. To improve its value as a proxy for counteradaptation, the measure was corrected for certain variables in the phenotyping set-up that could have influenced offspring production independent of the F3 genotype. We used the zeroinfl function of the Rpackage pscl (Zeileis et al. 2008) to fit the following full model by zero-inflated Poisson regression:

$n \_o f f s p r i n g \sim n \_n y m p h s+n \_w a s p s \_a d d e d+a l l \_r e-$ moved + any_found_dead + any_in_tube $\mid n \_n y m p h s+$ $n \_w a s p s \_a d d e d+a l l \_r e m o v e d+a n y \_f o u n d \_d e a d+$ any_in_tube

where $n \_o f f s p r i n g$ is the number of offspring wasps produced, $n \_n y m p h s$ is the number of aphid nymphs, i.e., potential hosts, provided, n_wasps_added is a factor describing whether one or two wasps were added to the aphid colony, all_removed is a factor describing whether all wasps could be recovered $24 \mathrm{~h}$ after adding them to the aphid colony, any_found_dead is a factor describing whether any of the wasps were dead after $24 \mathrm{~h}$, and any_in_tube is a factor describing whether any of the wasps were found in the tube rather than on the plant after $24 \mathrm{~h}$. Parameters before and after the I symbol are components of the Poisson and the zero-inflation part of the model, respectively. The or the R (resistant) genotype. Recombinant F2 males were backcrossed with females of the resistant population to produce semi-recombinant F3 females. Sister F3 females have identical chromosomes of paternal origin and are thus considered clonal sibships. Two hundred and fortyfour clonal sibships consisting of one to two sister F3 females were allowed to reproduce as virgins on a colony of symbiont-protected $(\mathrm{H}+)$ aphid hosts for phenotyping. Bar colors represent genomic regions originating from different parental populations and letters under sex symbols indicate the ploidy levels and genotypes.

full model was reduced to a minimal model by performing backwards elimination with the function be.zerofinl from the R-package mpath (Wang 2020). The final minimal model was:

$$
\text { n_offspring } \sim \text { n_nymphs }+ \text { all_removed }+ \text { any_in_tube | }
$$

n_wasps_added + any_in_tube.

Residuals of the minimal model were used as the corrected count phenotype for QTL mapping. We also assessed offspring presence presence/absence as an additional binary phenotype. Due to its simplicity, a binary phenotype may be less prone to environmental variation and more appropriate if counteradaptation is a Mendelian trait.

\section{DNA extraction and sequencing}

DNA extraction from $354 \mathrm{~F} 2$ males, $17 \mathrm{~F} 1$ females, and the two $\mathrm{P}$ individuals was performed adapting the LGCsbeadex Livestock D protocol (LGC Genomics, Berlin, Germany). In addition to these experimental individuals, 30 wasps from an asexual, isofemale line of L. fabarum (line CV17-84) were processed to quantify genotyping error. Due to their mode of reproduction and maintenance at small population size, CV17-84 individuals are expected to be genetically nearly identical. F2, F1, and P individuals and three pools of $10 \mathrm{CV} 17-84$ wasps each were crushed in liquid nitrogen prior to lysis. Extraction from individual samples was downscaled and included the following adaptations: lysis was done with PN buffer during $2 \mathrm{~h}$ at $60{ }^{\circ} \mathrm{C}$ with $1: 10$ protease solution, the lysate was incubated 
with binding mix during $20 \mathrm{~min}$ and elution was done at $60{ }^{\circ} \mathrm{C}$. Extraction from pooled samples was, besides doubling the amount of protease, done following the manual. DNA concentration of each sample was measured using a Spark 10 M Multimode Microplate Reader (Tecan, Switzerland). Quality of DNA obtained with the used protocols was tested on a Nanodrop spectrophotometer (Thermo Fisher Scientific, USA) and on agarose gels. ddRAD library preparation was adapted from the protocol by Peterson et al. (2012). Restriction enzymes MfeI and TaqI were used for double digestion of up to $50 \mathrm{ng}$ DNA per sample. After ligation of barcoded adapters to each individual sample, samples were combined in 12 pools with 24-36 samples each. Eleven pools contained one sample of $50 \mathrm{ng}$ DNA from the CV17-84 wasps and 23-35 other samples (F2, F1, or P). Fragment size selection was performed on each pool with AMPure XP beads (Beckman Coulter, USA) $(0.6 \times$ and $0.09 \times$ ) and followed by selection of biotinylated P2 adapters. This was followed by PCR with KAPA HiFi HotStart ReadyMix (Roche, Switzerland) to amplify DNA and add 12 different Illumina primers to identify pools. Pools were then purified and combined into a final library. Mean fragment size of the library was $606 \mathrm{bp}$, as measured with the 2200 TapeStation (Agilent, USA), which corresponds to a mean insert size of $470 \mathrm{bp}$. The library was sequenced in a single lane of an SP flow cell on an Illumina NovaSeq 6000 System with $2 \times 150 \mathrm{bp}$ paired-end sequencing (at Functional Genomic Center, Zürich). A total of 307.2 million paired-end reads were obtained from $\mathrm{P}, \mathrm{F} 1$, and F2 individuals and 11 CV17-84 control samples.

\section{Genotyping}

We used the dDocent pipeline (Puritz et al. 2014; Puritz et al. 2014) for genotyping. Reads were demultiplexed with the process_radtags function of the STACKS package (v 2.14, Catchen et al. 2013) with disabled filtering of degraded cut sites, which led to 304.2 million demultiplexed paired-end reads. BWA-MEM (v 0.7.17, Li and Durbin 2010) was used with default settings to map reads to the reference genome of L. fabarum (Lf_genome_V1.0.fa, Dennis et al. 2020). On average $( \pm \mathrm{SD}), 1.585( \pm 1.058)$ million reads were assigned per sample during demultiplexing. out of which an average of $81.66 \%$ were mapped and retained after filtering for mapping quality (Supplementary Table S1). We called 547,092 variants using freebayes (v 1.3.1, Garrison and Marth 2012) with the default settings from dDocent pipeline specifying population (corresponding to the generation P, F1, F2, or CV17-84) and ploidy of individuals. The VCF-file was then split into a dataset containing 355 haploid individuals, i.e., males (one P, 354 F2) and a dataset with 29 diploid individuals i.e., females (1 P, 11 CV17-84, $17 \mathrm{~F} 1$ ). The dataset with diploids was filtered following the dDocent filtering pipeline up until removing indels, retaining 2456 single-nucleotide polymorphisms (SNPs). The following changes were made to the tutorial: the minimum quality score $(-\min Q)$ was set to 20 , the minimum mean depth (-min-meanDP) was set to 10 , and the maximum mean depth $(-$ max-meanDP) was set to 400 . The haploid dataset was then transformed to allelic primitives and filtered to contain only the 2456 SNPs that were retained in the diploid dataset. The VCF files containing haploid and diploid samples were then transformed to SNP tables using samtools (v 1.9, Li et al. 2009) and custom bash scripts. A custom R-script was then used to filter the SNP tables and create an input file for linkage mapping with MSTmap (Wu et al. 2008). The retained SNPs are homozygous in the mother, biallelic among the two parent individuals, and known in both parent individuals. Additionally, we tested for segregation distortion, removing SNPs that deviate significantly from an allele frequency of $50 \%$ based on a chi-square test with Bonferroni-corrected false-discovery rate of $5 \%$. For each allele in each offspring (F2) male, alleles were recoded as "A" for maternal, "B" for paternal, and "U" for unknown. SNPs missing in $>50 \%$ of individuals and individuals with $>50 \%$ unknown genotypes were removed. The dataset used for linkage mapping contained 351 F2 individuals and 1838 SNPs of which 3 were removed by MSTmap internal filters leading to a final dataset of 1835 SNPs contained in the linkage map.

\section{Quantification of genotyping error}

Genotyping error rate was quantified by counting mismatches between the supposedly identical genotypes of 11 CV17-84 DNA samples that were sequenced as part of 11 different pools. The 1835 SNPs used for QTL mapping were used as a template to filter SNPs in the dataset with CV17-84 individuals with vcftools (-positions flag). A SNP table containing CV17-84 genotypes was then analyzed in $\mathrm{R}$ to quantify genotyping error. For each pair of CV17-84 samples, the proportion of genotype mismatches was counted and averaged over all comparisons to obtain an estimate of mean genotyping error. Unknown genotypes were not counted as mismatch. The mean percentage of pairwise mismatches among the 11 CV17-84 samples ranged from 0.8392 to $1.706 \%$ with an average of $1.207 \%$. The average mismatch measure was employed as an estimate for the genotyping error during analyses with R/qtl (Broman et al. 2003).

\section{Linkage map and QTL mapping}

Linkage mapping was performed with MSTmap (Wu et al. 2008) using the following settings: population_type $=D H$, distance_function $=k o s a m b i$, cut_off_p_value $=0.000001$, no_map_dist $=15.0$, no_map_size $=2$, missing_threshold $=0.25$, estimation_before_clustering $=n o$, detect_bad_data $=$ yes, objective function $=$ COUNT . The resulting 
Table 2 Observed offspring numbers from experimentally crossed resistant $(\mathrm{R})$ and susceptible (S) Lysiphlebus fabarum populations on Hamiltonella-protected aphid host.

\begin{tabular}{|c|c|c|c|c|c|c|c|}
\hline \multirow[t]{2}{*}{ Cross } & \multirow[t]{2}{*}{ Crosses with reproduction ${ }^{\mathrm{a}}$} & \multicolumn{3}{|c|}{ Female offspring } & \multicolumn{3}{|c|}{ Male offspring } \\
\hline & & Mean & SEM & Median & Mean & SEM & Median \\
\hline $\mathrm{RR} \times \mathrm{R}$ & $16 / 23$ & 9.87 & 1.98 & 8 & 5.74 & 1.71 & 5 \\
\hline $\mathrm{RR} \times \mathrm{S}$ & $13 / 27$ & 8.38 & 2.22 & 3 & 5.43 & 1.53 & 4 \\
\hline $\mathrm{SS} \times \mathrm{R}$ & $0 / 17$ & 0 & 0 & 0 & 0 & 0 & 0 \\
\hline $\mathrm{SS} \times \mathrm{S}$ & $0 / 18$ & 0 & 0 & 0 & 0 & 0 & 0 \\
\hline
\end{tabular}

${ }^{\mathrm{a}}$ For each cross, a wasp pair consisting of a male and a female was given $24 \mathrm{~h}$ for reproduction and oviposition on an aphid colony. distance matrix was processed with $\mathrm{R}$ to contain only marker locations, Linkage group (LG) ID, and map distance. The new linkage map was edited in order to use the same LG IDs and orientations as in the linkage map by Dennis et al. (2020).

Phenotype data, genotype data, and the new linkage map were merged with a custom $\mathrm{R}$ script to produce an input file for R/qtl (Broman et al. 2003). After reading the dataset with $\mathrm{R} / \mathrm{qtl}$, its cross type was transformed to recombinant-inbred by selfing (convert2riself function) because this expects no heterozygotes and genotype frequencies at 0.5 , which fits our crossing scheme. We tested for duplicated genotypes (>90\% similarity between individuals), checked for switched markers using the checkAlleles function, and plotted recombination fractions (Supplementary Fig. S1), none of which indicated any problems. Intermarker distance was estimated with the est.map function, setting map function to "kosambi" and tolerance to $10^{-4}$. The resulting map was used as new linkage map with $\mathrm{cM}$ as map unit. Conditional genotype probabilities were calculated at a step size of $0.1 \mathrm{cM}$. The scanone function was used to calculate logarithmic of the odds (LOD) scores over the genome using the default (EM) algorithm with nonparametric and binary model for the corrected count phenotype and the additional binary phenotype, respectively. Significance thresholds were calculated by conducting 1000 permutations and choosing a $5 \%$ cut-off corresponding to the significance threshold at an alpha of $5 \%$. The $95 \%$ approximate Bayes confidence interval was then calculated for the chromosome with significant LOD score. After simulating genotypes 1000 times with a step size of $0.1 \mathrm{cM}$ and pulling genotype probabilities at the peak LOD, the explained phenotypic variance was estimated with the fitqtl function.

\section{Candidate gene identification}

As RADseq loci are usually short and represent a small proportion of the genome, they are unlikely located in candidate genes themselves. The 95\% approximate Bayes confidence interval of the single significant QTL we identified includes all markers on scaffold tig00000002, upwards of 311,170 (bp). Thus, we considered tig00000002 from position 311,170 on as region for searching candidate genes. Gene annotations were retrieved from the recently published L. fabarum genome (Dennis et al. 2020). In addition, we identified putative venom and toxin genes in the L. fabarum genome in order to explore this function among candidate genes. To do so, we collected venom protein sequences from several parasitoid wasp species: Nasonia vitripennis (Danneels et al. 2010), Chelonus inanitus (Vincent et al. 2010), Microplitis demolitor (Burke and Strand 2014), Fopius arisanus (Geib et al. 2017), Diachasma alloeum (Tvedte et al. 2019), Cotesia congregata (Gauthier et al. 2021), Leptopilina boulardi, Leptopilina heterotoma (Goecks et al. 2013), and Aphidius ervi (Colinet et al. 2014); and retrieved candidate animal toxin proteins (7151 sequences) from the UniProt Animal Toxin Annotation Program database (UATdb, Jungo et al. 2012). These proteins were then matched to L. fabarum proteins by blastp (-e-value $<1 e-8,-m a x \_t a r g e t \_s e q s=10$, Camacho et al. 2009). This was combined with the 32 L. fabarum proteins identified as venoms by proteomic studies (Dennis et al. 2020).

\section{Results}

\section{Nature of the counteradaptation}

To determine the nature of the counteradaptation of $L$. fabarum to Hamiltonella-protected aphids, wasps from the $\mathrm{R}$ and $\mathrm{S}$ populations were crossed prior to assessing reproduction on $\mathrm{H}+$ aphids (Table 1). Out of $23 \mathrm{RR} \times \mathrm{R}, 21$ $\mathrm{RR} \times \mathrm{S}, 17 \mathrm{SS} \times \mathrm{R}$, and $18 \mathrm{SS} \times \mathrm{S}$ crosses, female offspring were observed exclusively in $16 \mathrm{RR} \times \mathrm{R}$ and $13 \mathrm{RR} \times \mathrm{S}$ crosses (Table 2 and Fig. 2). Mann-Whitney $U$ tests (followed by Bonferroni correction of $P$ values) showed significantly different numbers of female offspring in all four comparisons of crosses with RR mothers against crosses with SS mothers $(P<0.001)$ but not when comparing RR $\times$ $\mathrm{R}$ against $\mathrm{RR} \times \mathrm{S}$ crosses $(P=0.32$, Fig. $2 \mathrm{a})$. The fact that female offspring are only produced in crosses with $\mathrm{R}$ mothers is in line with $\mathrm{H} 3$, suggesting that counteradaptation is governed by the maternal genotype (Table 1). $\mathrm{H} 1$ and $\mathrm{H} 2$ 
A

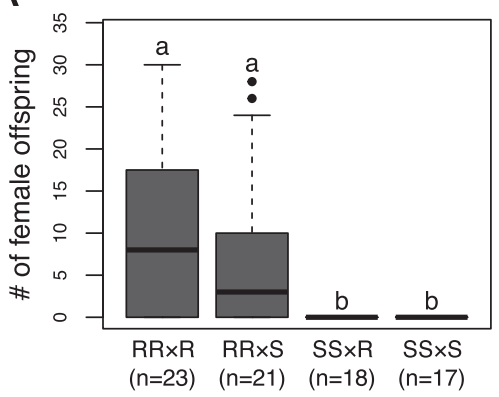

Fig. 2 Reproduction of crossed Lysiphlebus fabarum populations on Hamiltonella-protected aphids. A Female offspring numbers produced by the four different crosses between resistant (R) and susceptible (S) L. fabarum wasps on $\mathrm{H}+$ aphids. Numbers of offspring correspond to the reproduction of a male and a female wasp that were given $24 \mathrm{~h}$ to reproduce on a $\mathrm{H}+$ aphid colony. Letters indicate significant differences assessed by Mann-Whitney $U$ tests $(\mathrm{RR} \times \mathrm{R}$ vs $\mathrm{RR} \times \mathrm{S}: U=200, \mathrm{n} 1=23, \mathrm{n} 2=21, P=0.32 ; \mathrm{RR} \times \mathrm{R}$ vs $\mathrm{SS} \times \mathrm{R}: U=$

can be ruled out because both predict equal numbers of female offspring in $\mathrm{RR} \times \mathrm{S}$ and $\mathrm{SS} \times \mathrm{R}$ crosses (Table 1). A GLM test showed that sex ratios did not differ between $\mathrm{RR} \times \mathrm{S}$ and $\mathrm{RR} \times \mathrm{R}$ crosses (Fig. $2 \mathrm{~b}, P=0.730$ ). Therefore, no evidence was found for additional larval effects that would lead to differences in sex ratios between these crosses. In the second generation, 20 virgin (RS) female descendants from $10 \mathrm{RR} \times \mathrm{S}$ crosses were allowed to oviposit on $\mathrm{H}+$ aphids (Table 1). None of these RS females produced any offspring. This supports H3.2 and indicates that the counteradaptation is maternal and recessive. Additionally, it excludes that a cytoplasmic element is responsible, because the RS females had inherited the cytoplasm from their $\mathrm{H}+$-adapted RR mothers.

\section{Genotyping and linkage map construction}

From our backcross scheme (Fig. 1) starting with a single $\mathrm{P}$ generation cross, we obtained 17 F1 individuals that produced 354 F2 males (mapping population). The dataset used for linkage mapping and QTL analyses contained 351 F2 males and 1835 SNPs. The linkage map created from this dataset contained six LGs (Fig. 3), likely corresponding to the six chromosomes identified in L. fabarum by karyotyping (Belshaw and Quicke 2003). Estimation of intermarker distance with R/qtl yielded a total map size of $828.3 \mathrm{cM}$ with an average marker spacing of $0.4529 \mathrm{cM}$ and a maximum distance between markers on the same LG of $8.265 \mathrm{cM}$ (Table 3 and Supplementary Table S2). The 1835 SNPs were located across 352 scaffolds, representing $20.73 \%$ of the 1698 scaffolds from the $L$. fabarum reference genome assembly. The scaffolds included in the linkage map account for $59.94 \%$ (84.34 Mbp) of the $140.7 \mathrm{Mbp} L$.
B

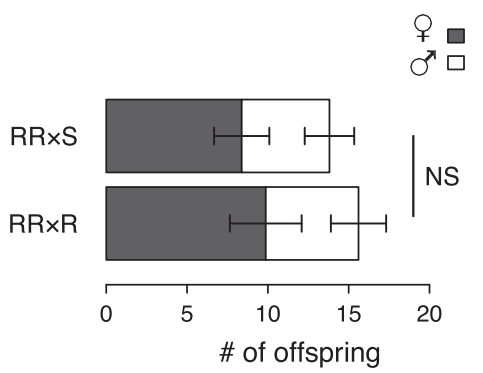

$59.5, \mathrm{n} 1=23, \mathrm{n} 2=18, P<0.001 ; \mathrm{RR} \times \mathrm{R}$ vs $\mathrm{SS} \times \mathrm{S}: U=63, \mathrm{n} 1=23$, $\mathrm{n} 2=17, P<0.001 ; \mathrm{RR} \times \mathrm{S}$ vs SS $\times \mathrm{R}: U=68, \mathrm{n} 1=21, \mathrm{n} 2=18, P<$ $0.001 ; \mathrm{RR} \times \mathrm{S}$ vs $\mathrm{SS} \times \mathrm{S}: U=72, \mathrm{n} 1=21, \mathrm{n} 2=17, P<0.001)$. B Comparison of offspring sex ratio between the two successful crosses. The stacked plot represent mean \pm SEM number of offspring individuals emerging per cross. GLM with quasibinomial error distribution and dispersion parameter taken to be 3.607 revealed no significant (NS) difference between sex ratios.

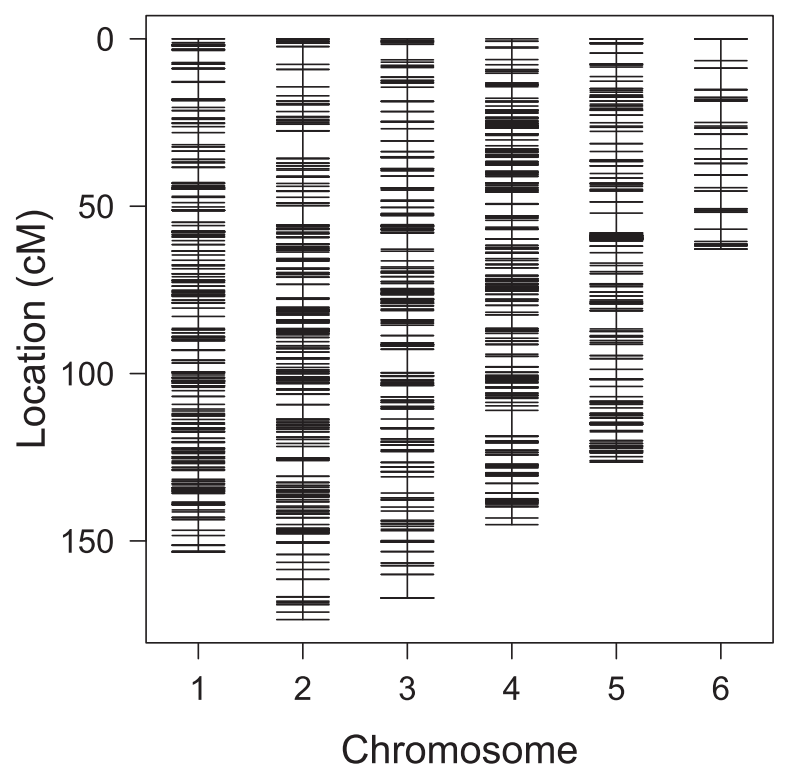

Fig. 3 Linkage map of the Lysiphlebus fabarum $\mathbf{F} 2$ cross. Repartition of the 1835 high-quality SNP markers over the six inferred linkage groups (LGs) representing a total size of $828.3 \mathrm{cM}$.

fabarum reference genome. Eight scaffolds were found to map on two different LGs (Supplementary Table S2) and are thus likely chimeric. Given the genome size and map length, the recombination rate of $L$. fabarum was estimated to be $5.887 \mathrm{cM} / \mathrm{Mbp}$. This recombination rate and the high marker density were both favorable to QTL detection. The linkage map resembles another recently constructed in terms of LG number, size, and included scaffolds (MattheyDoret et al. 2019, Dennis et al. 2020). However, our map provides a slight improvement compared to the previous 
Table 3 Genetic map summary.

\begin{tabular}{llclll}
\hline Linkage group & $\begin{array}{l}\text { Number of } \\
\text { markers }\end{array}$ & Length $(\mathrm{cM})$ & Length (Mbp) & $\begin{array}{l}\text { Average } \\
\text { spacing }(\mathrm{cM})\end{array}$ & $\begin{array}{l}\text { Maximum } \\
\text { spacing }(\mathrm{cM})\end{array}$ \\
\hline 1 & 368 & 153.354 & 17.5 & 0.417 & 5.121 \\
2 & 377 & 173.508 & 18.6 & 0.461 & 8.265 \\
3 & 304 & 167.050 & 15.3 & 0.551 & 7.035 \\
4 & 421 & 145.142 & 14.7 & 0.345 & 7.737 \\
5 & 304 & 126.455 & 13.6 & 0.417 & 5.965 \\
6 & 61 & 62.811 & 4.4 & 1.046 & 6.483 \\
Overall & 1835 & 828.322 & 84.3 & 0.452 & 8.265 \\
\hline
\end{tabular}

Length $(\mathrm{Mbp})$ represents the total length of scaffolds included in the linkage group/map.

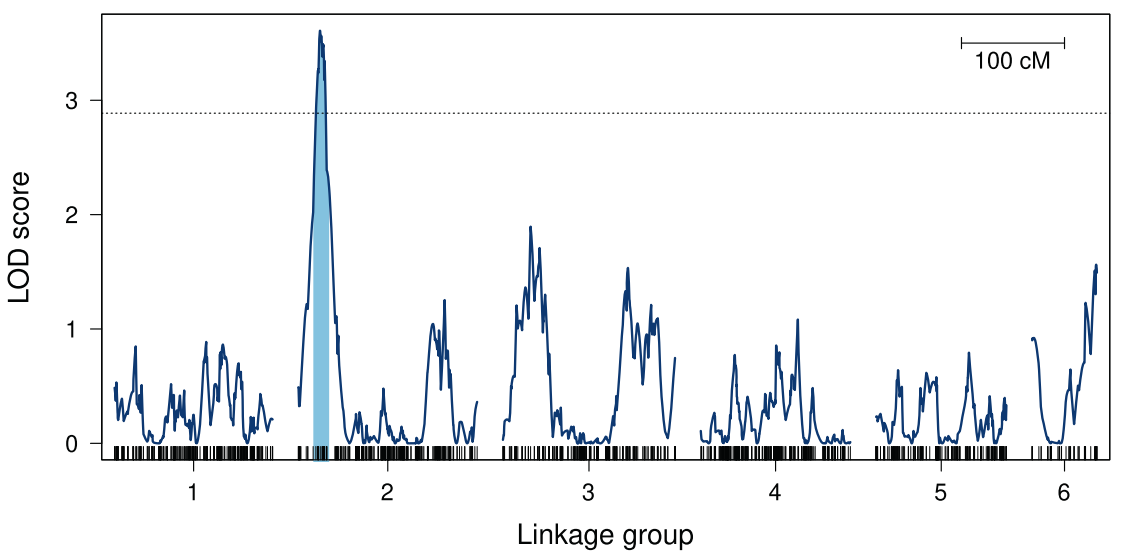

Fig. 4 QTL mapping. LOD scores of the nonparametric QTL model reveal a significant peak in LG 2. LOD scores were calculated at marker locations and between markers at $0.1 \mathrm{cM}$ intervals. The analysis was carried out with a corrected count phenotype representing counteradaptation. The horizontal dotted line shows the global

one in Dennis et al. (2020), which contained approximately $53 \%$ of the reference genome of L. fabarum (almost $60 \%$ for ours).

\section{Main QTL for counteradaptation}

By backcrossing F2 males with $\mathrm{R}$ population females, we obtained 244 F3 sibships for phenotyping. F3 females were tested for their ability to produce offspring on $\mathrm{H}+$ aphids. Three sibships were excluded due to missing F2 genotypes. Offspring were observed in 153 out of 241 sibships, which was significantly $>50 \%$ of the sibships $\left(\chi^{2}=17.53\right.$, df $=1$, $P<0.001)$. The average number of offspring per sibship $( \pm$ SEM) was $5.988( \pm 0.4472)$, across the range of $0-28$. The binomial part of the zero-inflation model showed that the probability to observe offspring in phenotyping colonies was affected by two variables. It was increased when two, instead of one, F3 females were added (n_wasps_added variable) and when both wasps had left the tubes within which they were added to the colony (any_in_tube variable). Offspring counts generally increased with aphid significance threshold for $P=0.05$, determined by permutation test $(N$ replicates $=1000)$. The blue region on LG 2 represents the $95 \%$ approximate Bayes confidence interval for the QTL located on this linkage group. Vertical ticks at the bottom show marker locations for each linkage group.

colony size (Supplementary Fig. S2 and Supplementary Table S3). The residuals from this model were taken as corrected count phenotypes for QTL mapping. We additionally used the binary variable of offspring presence/ absence as phenotype.

Two hundred and forty-one phenotype observations and 1835 SNPs were used for the QTL analysis with corrected count phenotypes. The significance threshold at an alpha of $5 \%$ was found to be at a LOD of 2.887. A single genomic region exhibited a LOD score above the significance threshold (Fig. 4). The peak LOD score of 3.608 occurred on LG 2 at $21 \mathrm{cM}(P=0.008)$. The marker with the maximum LOD score of 3.517 was located nearby at $21.72 \mathrm{cM}$. The region with LOD score above the significance threshold on LG 2 ranges from 17.2 to $26.5 \mathrm{cM}$. The $95 \%$ approximate Bayes confidence interval ranges from 15.1 to $29.3 \mathrm{cM}$. The single-QTL model could explain $7.25 \%$ of the observed phenotypic variation (Supplementary Fig. S3). Using the additional binary phenotype, a maximum LOD score of $1.733(P=0.544)$ was observed at $20.6 \mathrm{cM}$ on LG 2 , but no significant QTL was found (Supplementary Fig. S4). 
Table 4 Putative venom genes among the 120 candidate genes for counteradaptation to Hamiltonella located in the $95 \%$ Bayes confidence interval of the QTL identified in LG 2.

\begin{tabular}{llll}
\hline Gene ID & $\begin{array}{l}\text { Candidate } \\
\text { category }^{\mathrm{a}}\end{array}$ & Annotated function & $\begin{array}{l}\text { Distance from max. LOD } \\
\text { score marker }(\mathrm{Kbp})^{\mathrm{b}}\end{array}$ \\
\hline LF000355 & Toxin & Seminal metalloprotease 1 & 147.77 \\
LF000292 & Venom & Protein yellow & 708.93 \\
LF000293 & Venom & Chymotrypsin-1-like & 700.24 \\
LF000294 & Venom & Thioredoxin & 698.47 \\
LF000308 & Venom & Plasma membrane calcium-transporting & 487.21 \\
& & ATPase 2 & 324.01 \\
LF000319 & Venom & Neprilysin-like & 257.17 \\
LF000324 & Venom & Rho GAP 44-like & 418.39 \\
LF000401 & Venom & Eukaryotic peptide chain release factor & \\
& & GTP-binding subunit 3A & 444.03 \\
LF000408 & Venom & \\
& & Venom low-density lipoprotein receptor- & \\
\end{tabular}

${ }^{a}$ Venom and toxin: genes encoding for proteins matching parasitoid wasp venoms or animal toxins (determined by blastP).

${ }^{\mathrm{b}}$ The middle point of each gene was considered to calculate the distance from the maximum LOD score marker.

${ }^{c}$ Venom protein identified by mass spectrometric analysis of venom glands (Dennis et al. 2020).

\section{Candidate genes}

The QTL for counteradaptation to $H$. defensa identified on the LG 2 maps to positions on scaffold tig00000002 upwards of $311,170 \mathrm{bp}$ of the $L$. fabarum reference genome. This region corresponds to approximately $1.17 \mathrm{Mbp}$ of the scaffold tig00000002. According to the genome annotation, 120 coding genes are included in this region (Supplementary Table S4) and are thus candidate genes for the counteradaptation. Among these genes, nine genes may be of particular interest as they encode homolog proteins to venom proteins from other parasitoid wasp species or animal toxins (Table 4).

\section{Discussion}

This study represents the first attempt to investigate the genomic basis of the counteradaptation to symbiontconferred resistance in aphid parasitoids with a QTL approach. We found that counteradaptation is a recessive trait and determined by the maternal genotype. Further, we identified a QTL region that is associated with the counteradaptation. This was possible because we had at our disposal wasp lines diverging in their ability to parasitize symbiont-protected aphids from a previous experimental evolution study (Dennis et al. 2017).

Although we detected a single significant QTL associated with the counteradaptation to Hamiltonella-protected aphids on $L$. fabarum's LG 2, which would in principle be consistent with single-locus Mendelian inheritance, the genetic determination of the counteradaptation is unlikely to be that simple. With a single contributing locus, about $50 \%$ instead of the observed $36.51 \%$ of F3 sibships should have been unable to reproduce on $\mathrm{H}+$ aphids, likely leading to a clearer separation of phenotypes by the genotype (Supplementary Fig. S3), as it is the case, for example, for an insecticide-resistance QTL in bed bugs (Fountain et al. 2016). Moreover, a single-locus inheritance of the counteradaptation would likely have been detected in the additional analysis with offspring presence as binary phenotype, which detected no significant QTLs (Supplementary Fig. S4). The count-based phenotype may be more powerful at capturing loci associated with producing large numbers of offspring on $\mathrm{H}+$ aphids. Thus, the identified QTL is likely associated with the success rate of parasitizing $\mathrm{H}+$ aphids. Furthermore, the identified QTL may not represent the only region contributing to counteradaptation. With the countbased phenotype, we do indeed see other genomic regions with elevated LOD scores, e.g., on LG 3 (Fig. 4), but these were not statistically significant. Finally, because we did not find any evidence for a contribution of the offspring genotype in experiment 1 , our breeding design was not optimized to examine larval contributions acting in addition to the maternal counteradaptation we screened for. The existence of a larval contribution can thus not be ruled out.

We estimated that the QTL on LG 2 explains around $7.25 \%$ of the phenotypic variance in reproductive success on $\mathrm{H}+$ aphids. This percentage may seem low, but other QTL analyses in parasitoid wasps have also identified loci making modest contributions to the phenotypic variance. For example, QTLs identified by Pannebakker et al. (2011) explained 0.31 and $0.16 \%$ of the phenotypic variance in clutch size and sex ratio in $N$. vitripennis, respectively. 
Using a crossing design that involved the two species $N$. vitripennis and N. giraulti, Werren et al. (2016) found a QTL that explained $14 \%$ of the phenotypic variance in head width-to-head length ratio. In $C$. congregata, even 27.7 and $24.5 \%$ of the phenotypic variance observed in parasitism success and offspring number could be explained by individual QTLs (Benoist et al. 2020). It is possible that we underestimated the importance of the counteradaptation QTL, despite the size of our mapping population being close to the suggested range of 250-300 for QTL studies in $N$. vitripennis (Gadau et al. 2012), because the environmental variance $\left(V_{\mathrm{E}}\right)$ was certainly high. We observed a lot of variation in reproduction and even the presence of nonreproductive individuals from the pure $\mathrm{R}$ population in the first crossing experiment. This indicates that reproduction on $\mathrm{H}+$ aphids is a trait that is difficult to measure precisely, and the absence of reproduction on Hamiltonella-protected aphids does not necessarily reflect an absence of the ability to do so. We tried to reduce this variation somewhat by using the residuals of a zero-inflated GLM that corrected for some sources of $V_{\mathrm{E}}$, but the effect was limited. A possible improvement would consist in increasing the number of phenotyped sister F3 females to obtain a more accurate measure of their phenotype (Benoist et al. 2020). Additionally, a better control of the experimental set-up to reduce $V_{\mathrm{E}}$ (e.g., equal numbers of aphids available to parasitize) could contribute to a more precise measure of the counteradaptation trait.

Our observation that the counteradaptation of L. fabarum to the $H$. defensa strain H76 is mainly determined by the maternal genotype is somewhat surprising, given that the larvae have to complete their development in a host that contains toxin-producing symbionts, and it suggests that the relevant effectors are deposited at oviposition. They may comprise maternal contributions to the egg or belong to the manifold compounds females inject alongside the egg to suppress host immunity and thereby prime hosts for successful development of their offspring (Schmidt et al. 2001). We can thus speculate that the molecules injected by the wasp with its eggs may impair the protection conferred by Hamiltonella and its associated APSE phage, although it is still unknown if the counteradaptation indeed targets the bacteria directly or rather increases offspring tolerance of these bacteria. An important maternal mechanism making up the parasitism arsenal of many parasitoid wasps are symbiotic polydnavirus particles, virus-like particles, and venoms (Burke and Strand 2014). As the genome of $L$. fabarum is not known for having integrated a polydnavirus (Dennis et al. 2020), unlike other wasps from the Braconidae family (Gauthier et al. 2018), venoms are likely involved in the counteradaptation, as also suggested by transcriptome data in Dennis et al. (2017). The venoms of parasitoid wasps can serve various purposes as they induce a variety of physiological and behavioral changes in the host (Moreau and Asgari 2015). Even antimicrobial properties were shown in the case of the cysteine-rich peptide defensin-NV from $N$. vitripennis venom (Ye et al. 2010). Moreover, genes encoding wasp venom are rapidly evolving and diverse both among and within parasitoid wasp species (Cavigliasso et al. 2019), making them suitable targets for rapid evolution in an arms race between parasitoids and host-protective endosymbionts (Colinet et al. 2010). In the identified QTL candidate region, 9 genes out of 120 encode L. fabarum putative venoms, but they are not in the close vicinity of the peak with maximum LOD score (Supplementary Table 4). Indeed, among the closest candidate genes, none are predicted as putative venom genes. However, since the blast approach we used here identifies only parasitoid conserved venoms and the proteomic approach generally identifying a limited number of proteins (Dennis et al. 2020), we cannot exclude that some of the unknown proteins encoded by the candidate genes are $L$. fabarum specific venoms. Further tissue-specific analyses of venom glands would be needed to have an exhaustive overview of L. fabarum venoms.

The ability to parasitize Hamiltonella-protected aphids being a recessive trait-at least in the case of Hamiltonella strain H76-has implications for the evolution of parasitoid counteradaptation. Recessive alleles persist in a population for a long time even if the trait they determine is no longer under positive selection or becomes selected against (Agrawal and Whitlock 2012). Previous studies on this system found no obvious costs of parasitoid counteradaptation, but it was highly specific to the symbiont strain the parasitoids were confronted with (Rouchet and Vorburger 2014; Dennis et al. 2017). In a dynamic system with some turnover of symbiont strains, e.g., if fueled by negative frequency-dependent selection (Agrawal and Lively 2002; Kwiatkowski et al. 2012), prior adaptations may no longer be useful if a new symbiont strain becomes prevalent in the host population. They could even be detrimental if counteradaptations to different strains were mutually exclusive (currently unknown). Such turnover would, however, leave a legacy of prior adaptations in the form of "invisible" recessive alleles in the heterozygous state within the parasitoid population. They would form the substrate for adaptation from standing genetic variation (Barrett and Schluter 2008), ready to respond to future selection from defensive symbionts. This scenario is certainly consistent with the rapid speed of parasitoid counteradaptation observed under experimental evolution (Dion et al. 2011; Rouchet and Vorburger 2014; Dennis et al. 2017).

Combining crossing experiments with a QTL analysis, we showed that the counteradaptation of L. fabarum to Hamiltonella-protected aphids is a maternally determined trait, and we identified an associated QTL. This QTL 
explained only a low proportion of the phenotypic variance in the wasps' ability to parasitize $\mathrm{H}+$ aphids, a difficult-tomeasure trait that appears to suffer from a lot of environmental noise. The genomic region covered by this QTL contains several putative venom genes, potential candidates for targeting $H$. defensa and its bacteriophage, which are responsible for parasitoid resistance in aphids. Improvements in phenotyping and further studies (e.g., genome wide approaches) will be necessary to explain a larger proportion of the variance in wasp parasitism success on protected aphids and to narrow in on the responsible genes.

It would be premature to draw any general conclusions for the evolution of parasite counterdefenses against symbiont-mediated protection from our study, and the literature does not offer many other examples to compare it with. General conclusions may also be difficult for a more fundamental reason: the counteradaptations required for parasites to overcome symbiont-conferred resistance will depend on the mechanism by which the resistance is achieved. These mechanisms differ widely among defensive symbionts (reviewed in Gerardo and Parker 2014). One interesting example pertains to our very study system. Black bean aphids are also protected against Lysiphlebus parasitoids by tending ants, which can be regarded as macrobial protective symbionts (Stadler and Dixon 2005). The parasitoids' counteradaptation against these symbionts consists in the evolution of chemical mimicry. The cuticular hydrocarbons of Lysiphlebus wasps mimic those of their aphid hosts, preventing their removal from aphid colonies by ants (Liepert and Dettner 1996).

For bacterial defensive symbionts, one generality that has emerged is that host protection is often mediated by toxins (Ford and King 2016; Oliver and Perlman 2020), but the types of toxins may differ among symbiont strains, as shown for H. defensa (Degnan and Moran 2008; Oliver and Higashi 2019). In the experimental evolution study by Dennis et al. (2017), wasps were also evolved in the presence of a second strain of $H$. defensa (H402, carrying another APSE variant that encodes a different toxin), equally resulting in rapid and specific counteradaptation. The H402-adapted wasps were not included in the present study, but it would be interesting to compare the genomic basis underlying their counteradaptation to see whether the same or different genomic regions are involved. The involvement of different regions would support an idiosyncratic nature of parasitoid counteradaptation in response to particular defense mechanisms, and it could help to explain the observed specificity of $H$. defensa-L. fabarum interactions.

\section{Data availability}

Sequencing data related to this study can be found under the accession PRJEB39724 in the European Nucleotide
Archive (https://www.ebi.ac.uk/ena/browser/home). Count data, phenotypes, genotypes, and scripts used to perform the different analyses of this study are available at https://doi. org/10.5061/dryad.18931zcvz.

Acknowledgements We thank Frida Feijen (Eawag, Dübendorf) for sharing optimized DNA extraction protocol for small animals; Claudia Michel (ETH, Zürich) for providing adapted ddRAD protocol; and Jürgen Gadau (University of Münster) for advice during the planning of the study. The RAD libraries were generated in collaboration with the Genetic Diversity Centre (GDC) at ETH Zürich and sequencing was performed at the Functional Genomic Center Zürich (FGCZ). We thank Silvia Kobel and Aria Minder from The GDC for their support in the molecular laboratory. We finally thank Paula Rodriguez (Eawag, Dübendorf) for maintaining the wasp populations and carrying on the experimental evolution up to the time of this study. We used the supercomputing facilities from ETH Zürich (Euler Cluster). This work was funded by Eawag and the Swiss National Science Foundation (grant nr. 31003A_181969 to CV).

Funding Open Access funding provided by Lib4RI - Library for the Research Institutes within the ETH Domain: Eawag, Empa, PSI \& WSL.

\section{Compliance with ethical standards}

Conflict of interest The authors declare no competing interests.

Publisher's note Springer Nature remains neutral with regard to jurisdictional claims in published maps and institutional affiliations.

Open Access This article is licensed under a Creative Commons Attribution 4.0 International License, which permits use, sharing, adaptation, distribution and reproduction in any medium or format, as long as you give appropriate credit to the original author(s) and the source, provide a link to the Creative Commons license, and indicate if changes were made. The images or other third party material in this article are included in the article's Creative Commons license, unless indicated otherwise in a credit line to the material. If material is not included in the article's Creative Commons license and your intended use is not permitted by statutory regulation or exceeds the permitted use, you will need to obtain permission directly from the copyright holder. To view a copy of this license, visit http://creativecommons. org/licenses/by/4.0/.

\section{References}

Agrawal A, Lively CM (2002) Infection genetics: gene-for-gene versus matching-alleles models and all points in between. Evol Ecol Res 4:91-107

Agrawal AF, Whitlock MC (2012) Mutation load: the fitness of individuals in populations where deleterious alleles are abundant. Annu Rev Ecol Evol Syst 43:115-135

Anderson RM, May RM (1982) Coevolution of hosts and parasites. Parasitology 85:411-426

Barrett RDH, Schluter D (2008) Adaptation from standing genetic variation. Trends Ecol Evol 23:38-44

Bäumler AJ, Sperandio V (2016) Interactions between the microbiota and pathogenic bacteria in the gut. Nature 535:85-93

Belshaw R, Quicke DL (2003) The cytogenetics of thelytoky in a predominantly asexual parasitoid wasp with covert sex Genome 46:170-173 
Benoist R, Capdevielle-Dulac C, Chantre C, Jeannette R, Calatayud PA, Drezen J-M et al. (2020) Quantitative trait loci involved in the reproductive success of a parasitoid wasp. Mol Ecol 29:3476-3493

Bento G, Routtu J, Fields PD, Bourgeois Y, Du Pasquier L, Ebert D (2017) The genetic basis of resistance and matching-allele interactions of a host-parasite system: The Daphnia magna-Pasteuria ramosa model. PLoS Genet 13:e1006596

Broman KW, Wu H, Sen S, Churchill GA (2003) R/qtl: QTL mapping in experimental crosses. Bioinformatics 19:889-890

Burke GR, Strand MR (2014) Systematic analysis of a wasp parasitism arsenal. Mol Ecol 23:890-901

Camacho C, Coulouris G, Avagyan V, Ma N, Papadopoulos J, Bealer K et al. (2009) BLAST+: architecture and applications. BMC Bioinformatics 10:421

Catchen J, Hohenlohe PA, Bassham S, Amores A, Cresko WA (2013) Stacks: an analysis tool set for population genomics. Mol Ecol 22:3124-3140

Cavigliasso F, Mathé-Hubert H, Kremmer L, Rebuf C, Gatti J-L, Malausa $T$ et al. (2019) Rapid and differential evolution of the venom composition of a parasitoid wasp depending on the host strain. Toxins 11:629

Cayetano L, Vorburger C (2013) Genotype-by-genotype specificity remains robust to average temperature variation in an aphid/ endosymbiont/parasitoidsystem. J Evol Biol 26:1603-1610

Coffman KA, Burke GR (2020) Genomic analysis reveals an exogenous viral symbiont with dual functionality in parasitoid wasps and their hosts. PLoS Pathog 16:e1009069

Colinet D, Anselme C, Deleury E, Mancini D, Poulain J, AzémaDossat $C$ et al. (2014) Identification of the main venom protein components of Aphidius ervi, a parasitoid wasp of the aphid model Acyrthosiphon pisum. BMC Genomics 15:342

Colinet D, Schmitz A, Cazes D, Gatti J-L, Poirié M (2010) The origin of intraspecific variation of virulence in an eukaryotic immune suppressive parasite. PLoS Pathog 6:e1001206

Danneels EL, Rivers DB, De Graaf DC (2010) Venom proteins of the parasitoid wasp Nasonia vitripennis: recent discovery of an untapped pharmacopee. Toxins 2:494-516

Degnan PH, Moran NA (2008) Diverse phage-encoded toxins in a protective insect endosymbiont. Appl Environ Microbiol 74:6782-6791

Dennis AB, Ballesteros GI, Robin S, Schrader L, Bast J, Berghöfer J et al. (2020) Functional insights from the GC-poor genomes of two aphid parasitoids, Aphidius ervi and Lysiphlebus fabarum. BMC Genomics 21:376

Dennis AB, Patel V, Oliver KM, Vorburger C (2017) Parasitoid gene expression changes after adaptation to symbiont-protected hosts. Evolution 71:2599-2617

Dion E, Zélé F, Simon J-C, Outreman Y (2011) Rapid evolution of parasitoids when faced with the symbiont-mediated resistance of their hosts. J Evol Biol 24:741-750

Drezen J-M, Leobold M, Bézier A, Huguet E, Volkoff A-N, Herniou EA (2017) Endogenous viruses of parasitic wasps: variations on a common theme. Curr Opin Virol 25:41-48

Dubuffet A, Dupas S, Frey F, Drezen J, Poirie M, Carton Y (2007) Genetic interactions between the parasitoid wasp Leptopilina boulardi and its Drosophila hosts. Heredity 98:21-27

Flor H (1956) The complementary genic systems in flax and flax rust Adv Genet 8:29-54

Forbes AA, Bagley RK, Beer MA, Hippee AC, Widmayer HA (2018) Quantifying the unquantifiable: why Hymenoptera, not Coleoptera, is the most speciose animal order. BMC Ecol 18:21

Ford SA, King KC (2016) Harnessing the power of defensive microbes: evolutionary implications in nature and disease control. PLoS Pathog 12:e1005465
Fountain T, Ravinet M, Naylor R, Reinhardt K, Butlin RK (2016) A linkage map and QTL analysis for pyrethroid resistance in the bed bug Cimex lectularius. G3 Genes Genomes Genet 6:4059-4066

Frank SA (1997) Recognition and polymorphism in host-parasite genetics. In: Hamilton WD, Howard JC (eds) Infection, polymorphism and evolution. Springer, Dordrecht, p 13-23.

Gadau J, Pietsch C, Beukeboom LW (2012) Quantitative trait locus analysis in haplodiploid Hymenoptera. In: Rifkin SA (ed) Quantitative trait loci (QTL): methods and protocols, methods in molecular biology. Humana Press, Totowa, NJ, p 313-328

Garrison E, Marth G (2012) Haplotype-based variant detection from short-read sequencing. Preprint at https://arxiv.org/abs/1207.3907

Gauthier J, Boulain H, van Vugt JJFA, Baudry L, Persyn E, Aury J-M et al. (2021) Chromosomal scale assembly of parasitic wasp genome reveals symbiotic virus colonization. Commun Biol $4: 1-15$

Gauthier J, Drezen J-M, Herniou EA (2018) The recurrent domestication of viruses: major evolutionary transitions in parasitic wasps. Parasitology 145:713-723

Geib SM, Liang GH, Murphy TD, Sim SB (2017) Whole genome sequencing of the braconid parasitoid wasp Fopius arisanus, an important biocontrol agent of pest tepritid fruit flies. G3 Genes Genomes Genet 7:2407-2411

Gerardo NM, Parker BJ (2014) Mechanisms of symbiont-conferred protection against natural enemies: an ecological and evolutionary framework. Curr Opin Insect Sci 4:8-14

Godfray HCJ (1994) Parasitoids: behavioral and evolutionary ecology. Princeton University Press, Princeton

Goecks J, Mortimer NT, Mobley JA, Bowersock GJ, Taylor J, Schlenke TA (2013) Integrative approach reveals composition of endoparasitoid wasp venoms. PLoS ONE 8:e64125

Goodrich-Blair H, Clarke DJ (2007) Mutualism and pathogenesis in Xenorhabdus and Photorhabdus: two roads to the same destination. Mol Microbiol 64:260-268

Haine ER (2008) Symbiont-mediated protection. Proc R Soc B Biol Sci 275:353-361

Henry LM, Maiden MCJ, Ferrari J, Godfray HCJ (2015) Insect life history and the evolution of bacterial mutualism. Ecol Lett 18:516-525

Heraty J (2009) Parasitoid biodiversity and insect pest management. Wiley, New York

Itioka T, Inoue T (1996) Density-dependent ant attendance and its effects on the parasitism of a honeydew-producing scale insect, Ceroplastes rubens. Oecologia 106:448-454

Jaenike J (2012) Population genetics of beneficial heritable symbionts. Trends Ecol Evol 27:226-232

Jungo F, Bougueleret L, Xenarios I, Poux S (2012) The UniProtKB/ Swiss-Prot Tox-Prot program: a central hub of integrated venom protein data. Toxicon 60:551-557

Kwiatkowski M, Engelstädter J, Vorburger C (2012) On genetic specificity in symbiont-mediated host-parasite coevolution. PLoS Comput Biol 8:e1002633

Li H, Durbin R (2010) Fast and accurate long-read alignment with Burrows-Wheeler transform. Bioinformatics 26:589-595

Li H, Handsaker B, Wysoker A, Fennell T, Ruan J, Homer N et al. (2009) The Sequence Alignment/Map format and SAMtools. Bioinformatics 25:2078-2079

Liepert C, Dettner K (1996) Role of cuticular hydrocarbons of aphid parasitoids in their relationship to aphid-attending ants. J Chem Ecol 22:695-707

Martinez AJ, Ritter SG, Doremus MR, Russell JA, Oliver KM (2014) Aphid-encoded variability in susceptibility to a parasitoid. BMC Evol Biol 14:127

Matthey-Doret C, Van Der Kooi CJ, Jeffries DL, Bast J, Dennis AB, Vorburger C, Schwander T (2019) Mapping of multiple 
complementary sexdetermination loci in a parasitoid wasp Genome Biol Evol 11:2954-2962

McLean AHC, Godfray HCJ (2015) Evidence for specificity in symbiont-conferred protection against parasitoids. Proc R Soc B Biol Sci 282:20150977

Moran NA, Russell JA, Koga R, Fukatsu T (2005) Evolutionary relationships of three new species of enterobacteriaceae living as symbionts of aphids and other insects. Appl Environ Microbiol 71:3302-3310

Moreau SJM, Asgari S (2015) Venom proteins from parasitoid wasps and their biological functions. Toxins 7:2385-2412

Oliver KM, Degnan PH, Hunter MS, Moran NA (2009) Bacteriophages encode factors required for protection in a symbiotic mutualism. Science 325:992-994

Oliver KM, Higashi CH (2019) Variations on a protective theme: Hamiltonella defensa infections in aphids variably impact parasitoid success. Curr Opin Insect Sci 32:1-7

Oliver KM, Moran NA, Hunter MS (2005) Variation in resistance to parasitism in aphids is due to symbionts not host genotype. Proc Natl Acad Sci USA 102:12795-12800

Oliver KM, Noge K, Huang EM, Campos JM, Becerra JX, Hunter MS (2012) Parasitic wasp responses to symbiont-based defense in aphids. BMC Biol 10:11

Oliver KM, Perlman SJ (2020) Toxin-mediated protection against natural enemies by insect defensive symbionts. Adv Insect Physiol 58:277-316

Oliver KM, Russell JA, Moran NA, Hunter MS (2003) Facultative bacterial symbionts in aphids confer resistance to parasitic wasps. Proc Natl Acad Sci USA 100:1803-1807

Oliver KM, Smith AH, Russell JA (2014) Defensive symbiosis in the real world - advancing ecological studies of heritable, protective bacteria in aphids and beyond. Funct Ecol 28:341-355

Pannebakker BA, Watt R, Knott SA, West SA, Shuker DM (2011) The quantitative genetic basis of sex ratio variation in Nasonia vitripennis: a QTL study. J Evol Biol 24:12-22

Pennacchio F, Strand MR (2006) Evolution of developmental strategies in parasitic Hymenoptera. Annu Rev Entomol $51: 233-258$

Peterson BK, Weber JN, Kay EH, Fisher HS, Hoekstra HE (2012) Double Digest RADseq: an inexpensive method for de novo SNP discovery and genotyping in model and non-model species. PLoS ONE 7:e37135

Puritz JB, Hollenbeck CM, Gold JR (2014) dDocent: a RADseq, variant-calling pipeline designed for population genomics of nonmodel organisms. PeerJ 2:e431

Puritz JB, Matz MV, Toonen RJ, Weber JN, Bolnick DI, Bird CE (2014) Demystifying the RAD fad. Mol Ecol 23:5937-5942

Quicke DLJ (2015) The Braconid and Ichneumonid parasitoid wasps: biology, systematics, evolution and ecology. Wiley, New York

Rouchet R, Vorburger C (2012) Strong specificity in the interaction between parasitoids and symbiont-protected hosts. J Evol Biol 25:2369-2375

Rouchet R, Vorburger C (2014) Experimental evolution of parasitoid infectivity on symbiont-protected hosts leads to the emergence of genotype specificity. Evolution 68:1607-1616

Rossbacher S, Vorburger C (2020) Prior adaptation of parasitoids improves biological control of symbiont-protected pests. Evolutionary Applications 13:1868-1876
Sandrock C, Gouskov A, Vorburger C (2010) Ample genetic variation but no evidence for genotype specificity in an all-parthenogenetic host-parasitoid interaction. J Evol Biol 23:578-585

Sasaki A (2000) Host-parasite coevolution in a multilocus gene-forgene system. Proc R Soc Lond B Biol Sci 267:2183-2188

Schlötterer C, Kofler R, Versace E, Tobler R, Franssen SU (2015) Combining experimental evolution with next-generation sequencing: a powerful tool to study adaptation from standing genetic variation. Heredity 114:431-440

Schmid M, Sieber R, Zimmermann Y-S, Vorburger C (2012) Development, specificity and sublethal effects of symbiont-conferred resistance to parasitoids in aphids. Funct Ecol 26:207-215

Schmidt O, Theopold U, Strand M (2001) Innate immunity and its evasion and suppression by hymenopteran endoparasitoids. Bioessays 23:344-351

Stadler B, Dixon AFG (2005) Ecology and evolution of aphid-ant interactions. Annu Rev Ecol Evol Syst 36:345-372

R Core Team (2018) R: a language and environment for statistical computing. R Foundation for Statistical Computing, Vienna, Austria.

Tvedte ES, Walden KKO, McElroy KE, Werren JH, Forbes AA, Hood GR et al. (2019) Genome of the parasitoid wasp Diachasma alloeum, an emerging model for ecological speciation and transitions to asexual reproduction. Genome Biol Evol 11:2767-2773

Van Valen L (1977) The red queen. Am Nat 111:809-810

Vincent B, Kaeslin M, Roth T, Heller M, Poulain J, Cousserans F et al. (2010) The venom composition of the parasitic wasp Chelonus inanitus resolved by combined expressed sequence tags analysis and proteomic approach. BMC Genomics 11:693

Vorburger C (2014) The evolutionary ecology of symbiont-conferred resistance to parasitoids in aphids. Insect Sci 21:251-264

Vorburger C, Perlman SJ (2018) The role of defensive symbionts in host-parasite coevolution. Biol Rev 93:1747-1764

Vorburger C, Rouchet R (2016) Are aphid parasitoids locally adapted to the prevalence of defensive symbionts in their hosts? BMC Evol Biol 16:271

Vorburger C, Sandrock C, Gouskov A, Castañeda LE, Ferrari J (2009) Genotypic variation and the role of defensive endosymbionts in an all-parthenogenetic host-parasitoid interaction. Evolution 63:1439-1450

Wang Z (2020) mpath: regularized linear models, R package version $0.3-23$

Werren JH, Cohen LB, Gadau J, Ponce R, Baudry E, Lynch JA (2016) Dissection of the complex genetic basis of craniofacial anomalies using haploid genetics and interspecies hybrids in Nasonia wasps. Dev Biol 415:391-405

Windsor DA (1998) Controversies in parasitology, most of the species on earth are parasites. Int J Parasitol 28:1939-1941

Woolhouse MEJ, Webster JP, Domingo E, Charlesworth B, Levin BR (2002) Biological and biomedical implications of the coevolution of pathogens and their hosts. Nat Genet 32:569-577

Wu Y, Bhat PR, Close TJ, Lonardi S (2008) Efficient and accurate construction of genetic linkage maps from the minimum spanning tree of a graph. PLoS Genet 4(10):e1000212

Ye J, Zhao H, Wang H, Bian J, Zheng R (2010) A defensin antimicrobial peptide from the venoms of Nasonia vitripennis. Toxicon 56:101-106

Zeileis A, Kleiber C, Jackman S (2008) Regression models for count data in R. J Stat Softw 27:1-25 